\title{
Disinhibited Abuse of Othered Communities by Second-Screening Audiences
}

\author{
Mark Doughty, Shaun \\ Lawson, Conor Linehan, \\ Duncan Rowland \\ University of Lincoln, \\ Lincoln, UK. \\ mdoughty@lincoln.ac.uk
}

\author{
Lucy Bennett \\ Independent Researcher \\ Cardiff, UK. \\ bennettlucyk@gmail.com
}

\begin{abstract}
Second-screening and live-tweeting alongside broadcast television generates new concerns with respect to online abuse. We present an investigation into the nature of Twitter-facilitated second-screening posts relating to Thelma's Gypsy Girls, one of a series of controversial documentary programmes portraying the Irish Traveller community that have recently been aired by the UK publicservice television broadcaster Channel 4. Sentiment analysis highlighted the general negativity of these posts whilst a detailed thematic inquiry revealed the often abusive and aggressive messages aimed directly at the community and individuals portrayed in the broadcast material. We discuss why users might be susceptible to exhibiting these behaviours, and the implications for the broadcast industry, and social TV designers and developers.
\end{abstract}

\section{Author Keywords}

Television; Social TV; Social Media; Live-tweeting; Online Disinhibition; Second Screening; Abuse.

\section{ACM Classification Keywords}

H.5.m. Information interfaces and presentation (e.g., HCI): Miscellaneous.

\section{INTRODUCTION}

The viewing of broadcast television has always given rise to the concept of 'backchannel' communications [1]. Traditionally this has, perhaps, has taken the form of sharing private comments about a broadcast show with partners and family, while watching together in the living room, or discussing the show the following day with work colleagues or friends. In each of these instances, we are naturally drawn into the sharing of our opinions regarding the media we are co-consuming as well as the characters and individuals portrayed in that media. The deliberate activity of simultaneously watching a broadcast television show whilst engaging in online discussion about its content, variously known as 'co-viewing' [10], 'second-screening

Permission to make digital or hard copies of all or part of this work for personal or classroom use is granted without fee provided that copies are not made or distributed for profit or commercial advantage and that copies bear this notice and the full citation on the first page. To copy otherwise, or republish, to post on servers or to redistribute to lists, requires prior specific permission and/or a fee. Request permissions from permissions@acm.org.

TVX'14, June 25 - June 27, 2014, Newcastle, UK.

Copyright 2014 ACM 978-1-4503-2838-8/14/06...\$15.00.
[11], or 'live-tweeting' [23], is a widespread and rapidly increasing phenomena. Broadly positioned within the more established research field of social TV [8, 12], this stillemergent model of socially-experienced television incorporates the use of a 'second screen' through which an individual can interact with other viewers, who together comprise a wider, distributed co-interested audience. Online second-screen activity therefore expands television-related discussion far beyond co-located family, friends and colleagues into a much wider, networked group, public, or audience.

The current predominant, user-driven, approach to secondscreening can be considered a loosely-coupled model of interaction as it is enabled by general-purpose social media platforms such as Twitter [23]. In this model, any shared statements (e.g. tweets), any interactions between viewers (e.g. re-tweets, (RTs) and replies), and any client software itself (e.g. the Twitter app on a tablet device) are independent of the broadcast content, and of the control of the broadcaster. In the case of Twitter, live ad-hoc online audiences form through the shared and negotiated use of \#hashtags. Using Twitter's search function to stream tweets containing the hashtag \#Sherlock, for instance, allows easy, and instantaneous, access to live discussion about a particular broadcast TV show, generated by an ad hoc group of Twitter users who do not need to be connected through previously established 'follower' networks (see [6] for discussion). Of course, not all tweets about a broadcast will contain the same, consistent, hashtag; some tweets will contain alternative hashtags, such as the name of the TV Channel (e.g. $\# B B C 1$ ), whilst others may not contain a hashtag at all. The use of hashtags however offers enormous power and flexibility to the user; moreover, the experiences that they facilitate remain independent of broadcasters' control.

Second-screen experiences can also be facilitated by bespoke 'companion apps' that deliver additional digital content and filtered social media streams to a second-screen device. This, more tightly coupled, model of secondscreening allows broadcasters to stage-manage the user experience and is increasingly being pursued by that industry. However the orchestrated use of \#hashtags by broadcasters is also becoming apparent. A case of this has 
arisen around the controversial documentary programme Benefits Street recently aired by the British public-service television broadcaster Channel 4 where it has been alleged ${ }^{1}$ that viewers were deliberately reminded of the relevant hashtag (\#BenefitsStreet) at carefully planned moments within each episode in order to intensify online discussion and, perhaps, arouse further controversy.

The broadcast of Benefits Street has provoked intense UK media discussion, and criticism, primarily because of the nature of its portrayal of a particular community whose lives, allegedly funded by state-benefits, have been placed in public view on prime-time, free-to-air television. Observations of second-screen discussions on Twitter using \#BenefitsStreet, have also highlighted the particularly high levels of antipathy, anger and abuse directed at the community, and individuals, portrayed within the programme $^{2}$. Such discussion is reflective of recent instances in the UK where discriminatory, abusive and emotionally harmful tweets, which clearly refer to a specific individual or group/community, have received high-profile media attention, resulting, in some cases, in criminal prosecutions ${ }^{3}$. Despite the obvious public nature of the Twitter timeline, and the clear possibility that the owner of any Twitter account can be identified and, potentially, prosecuted, the online abuse of individuals and groups/communities remains an everyday occurrence ${ }^{4}$. In response to this, Guitton [14] recently called for a concerted research effort to understand the 'dark side of social media' and poses questions including: "Why do individuals display such aggressive behaviors toward people they don't know via social media?" and "Why do temporary communities emerge to systematically attack and harass those who appear as "weaker" on social media?".

We believe that second-screening and live-tweeting alongside broadcast television represents a particularly problematic area with respect to abuse and social media. Broadcasters increasingly rely on "reality" TV to garner audience share; these productions often emphasize how individuals, groups and communities can be extraordinary

\footnotetext{
${ }^{1}$ As discussed in "Benefits Street - poverty porn, or just the latest target for pent-up British fury?" Article in The Guardian (12 Jan 2014) http://www.theguardian.com/commentisfree/2014/jan/12/benefits-streetpoverty-porn-british-fury

${ }^{2}$ See "Benefits Street Twitter reactions: The angry, the idiotic and the defensive" Article in The Independent (8 Jan 2014) http://www. independent.co.uk/arts-entertainment/tv/features/benefits-street-twitterreactions-the-angry-the-idiotic-and-the-defensive-9046806.html

${ }^{3}$ For instance "Twitter 'trolls' Isabella Sorley and John Nimmo jailed for abusing feminist campaigner Caroline Criado-Perez" Article in the Independent (24 January 2014) http://www.independent.co.uk/news/uk/ crime/twitter-trolls-isabella-sorley-and-john-nimmo-jailed-for-abusingfeminist-campaigner-caroline-criadoperez-9083829.html

${ }^{4}$ For instance: "Sky Sports condemns 'unacceptable and offensive abuse' of Beth Tweddle" Article in The Guardian (21 Jan 2014) http://www. theguardian.com/sport/2014/jan/21/sky-sports-abuse-beth-tweddle
}

but negatively different [28] to general society. This othering [21] is not only evident in many broadcast productions but also anecdotally continues in the parallel and subsequent online discussion by second-screen audiences. This raises very broad questions around whether social media is being used, unwittingly or otherwise, to amplify, exaggerate, legitimize, or else facilitate the othering or marginalization of groups or communities. Additionally therefore, what is the effect of this secondscreening abuse in offline settings and society in the large, and what are the implications of this phenomenon for the broadcast and social TV industries? For the TVX community there are initial questions around whether there is indeed clear empirical evidence for such abuse and, if so (to return to Guitton's questions), why do people feel that they are able to post such abuse with impunity in online settings? Deeper understanding of these issues would allow for subsequent informed debate around the larger societal issues and industry implications.

The work presented in this paper focuses upon an analysis of the posts generated by second-screening viewers of Thelma's Gypsy Girls, one of a series of broadcast documentaries portraying the Irish traveller community in the UK and also recently aired by Channel 4. Like Benefits Street, this series of programmes has also provoked intense media discussion and observations of othering have been made of the second-screen discussions [28]. We investigate the extent to which the second-screening posts around Thelma's Gypsy Girls exhibit positive or negative sentiment, and, through a qualitative analysis, determine the themes present in the posts. Our discussion then focuses on possible explanations for the negative and abusive posting that we found and, in particular, whether there was evidence of online disinhibition [24] being displayed. Finally, we discuss the implications of this secondscreening behaviour for the TVX research community and the broadcast industry.

\section{BACKGROUND}

For the purposes of this study it is useful to define what constitutes abuse when considering online interactions. Abuse and threats of violence are a criminal act, whether these are made in face to face $(\mathrm{F} 2 \mathrm{~F})$ or online settings. Jay and Janschewitz [16] propose that offensive language consists of vulgar, pornographic and hateful terms. If this offensive language is directed at an individual, group or culture through an online interaction then we consider this an abusive communication. Cyber-bullying [19, 29] is also a relevant term within this context. Constituting the writing and posting of electronic messages to facilitate deliberate harassment or threat to another individual or group, it is an important aspect of the behaviour to consider when reviewing the implications for the work presented here. Thus offensive text aimed at an individual, ethnic or minority group, whether or not within the intention of the writer to offend is classed as abusive and harmful. 


\section{Explanations for Online Abuse}

A common, broad, finding of computer-mediated communication is that users can behave differently or say things online which they would not usually say in F2F settings. This phenomenon, known as the online disinhibition effect $[24,18]$, is understood to be as a result of the less socially-constraining nature of online communications [7] possibly resulting from the asynchronous or anonymous characteristics therein. The nature of online disinhibition can be described [18] as behaviour which is characterized by an apparent reduction in concerns for self-presentation and the judgment of others. Online disinhibition can manifest itself in different forms relating to what Suler [24] calls 'benign' factors such as self disclosure, involving fears, wishes and emotions, as well as more 'toxic' factors such as rude language, anger, hatred and threats. Both positive and benign disinhibition can be observed in Twitter communications [9].

One negative dimension of online disinhibition is the concept of 'flaming' [25]. While Kiesler, as cited by Joinson [18], categorizes flaming as messages including impolite statements, swearing, exclamations or the use of superlatives, O'Sullivan and Flanagin [25] highlight the problem of the contextual ambiguity of flame messages and the difficulty in interpreting them when viewed from one of the three perspectives of sender, receiver and third party observer. However, while these ambiguities are present in Twitter, the contextualising of a posting to a particular individual, group or topic using hashtag classifiers and user names can potentially reduce contextual ambiguity and result in more targeted and clearly abusive messages.

\section{Thelma's Gypsy Girls}

The documentary series Thelma's Gypsy Girls forms the focus of the second-screening activity investigated in this paper. It is a sequel to a previous documentary series Big Fat Gypsy Weddings; both series were produced by Firecracker Films and broadcast in the UK by Channel 4 . Each series has caused controversy due to their alleged negative depiction of the Irish Traveller community [28]. Thelma's Gypsy Girls follows the activities of a group of young teenage women from this community as they undertake an apprenticeship with a specialist dressmaker (the eponymous "Thelma"). Viewing figures for Thelma's Gypsy Girls averaged two million viewers per episode [2] which placed it consistently in top three shows viewed on Channel 4 during its run from 8th July to 12th August 2012.

\section{DATA COLLECTION}

Using the public Twitter stream API, we gathered 1,382 tweets from 839 unique users that contained the hashtag \#ThelmasGypsyGirls on 22nd July 2012 between 21:00GMT and 22:00GMT: the broadcast time for episode three of the show during its original run. The broadcaster, Channel 4, publicized this hashtag on screen before, and periodically during, the show. It is self-evident that there will have been tweets posted about the show which did not contain this hashtag; however by using this tag, we can be very certain that the poster was intending the tweet to be about the show. Retweeted messages (or simply retweets, RTs) potentially indicate the existence of conversation [4], community structure [11], and affirmations of sentiment; in this study we were primarily interested in original, unique posts and the individuals' motivations for making those posts. Therefore the $207 \mathrm{RT}$ messages in the dataset were set aside for separate analysis, leaving a main corpus of 1175 tweet messages. There were no incidences of tweets in a language other than English that required removal prior to analysis.

In order to determine how different our Thelma's Gypsy Girls corpus was from messages otherwise posted commonly on Twitter, a sample of random tweets was gathered using the public stream API. By collecting the messages at approximately the same time as the broadcast, and filtering the sample to remove non English tweets and RTs, a corpus of 7,902 general tweets was obtained. In order to compare like sample sizes, every 6th tweet was removed from this sample of random tweets to create a final general sample of 1,317 tweets.

\begin{tabular}{|c|c|c|}
\hline Corpus & $\begin{array}{c}\text { Negative } \\
\text { Sentiment } \\
\text { Mean } \\
\text { (Standard } \\
\text { Deviation) }\end{array}$ & $\begin{array}{c}\text { Positive } \\
\text { Sentiment Mean } \\
\text { (Standard } \\
\text { Deviation) }\end{array}$ \\
\hline $\begin{array}{c}\text { \#ThelmasGypsy } \\
\text { Girls } \\
(\mathrm{n}=1175)\end{array}$ & $-1.65(1.05)$ & $1.51(0.73)$ \\
\hline $\begin{array}{c}\text { General tweets } \\
(\mathrm{n}=1317)\end{array}$ & $-1.23(0.66)$ & $1.52(0.68)$ \\
\hline $\begin{array}{c}\text { \#ThelmasGypsy } \\
\text { Girls (RTs) } \\
(\mathrm{n}=207)\end{array}$ & $-1.98(1.12)$ & $1.38(0.63)$ \\
\hline
\end{tabular}

Table 1. Comparison of sentiment strengths across different tweet corpuses.

\section{SENTIMENT ANALYSIS}

A sentiment analysis of the collected tweets was undertaken to make a judgment on the degree of positive or negative opinion, or attitude, encoded in the tweet posts about Thelma's Gypsy Girls. Sentiment analysis is the computational treatment of opinion, sentiment and subjectivity in texts and encompasses many foci of study; however, for the purposes this work, we were interested in assessing the sentiment polarity of the text. Any written text may contain a mix of positive, negative or neutral sentiment, and there may also be differing strengths of the sentiment expressed [26]. The levels of sentiment in Twitter postings should not be exaggerated in their importance [27]; nevertheless, the levels investigated in this study provide a useful initial context. 

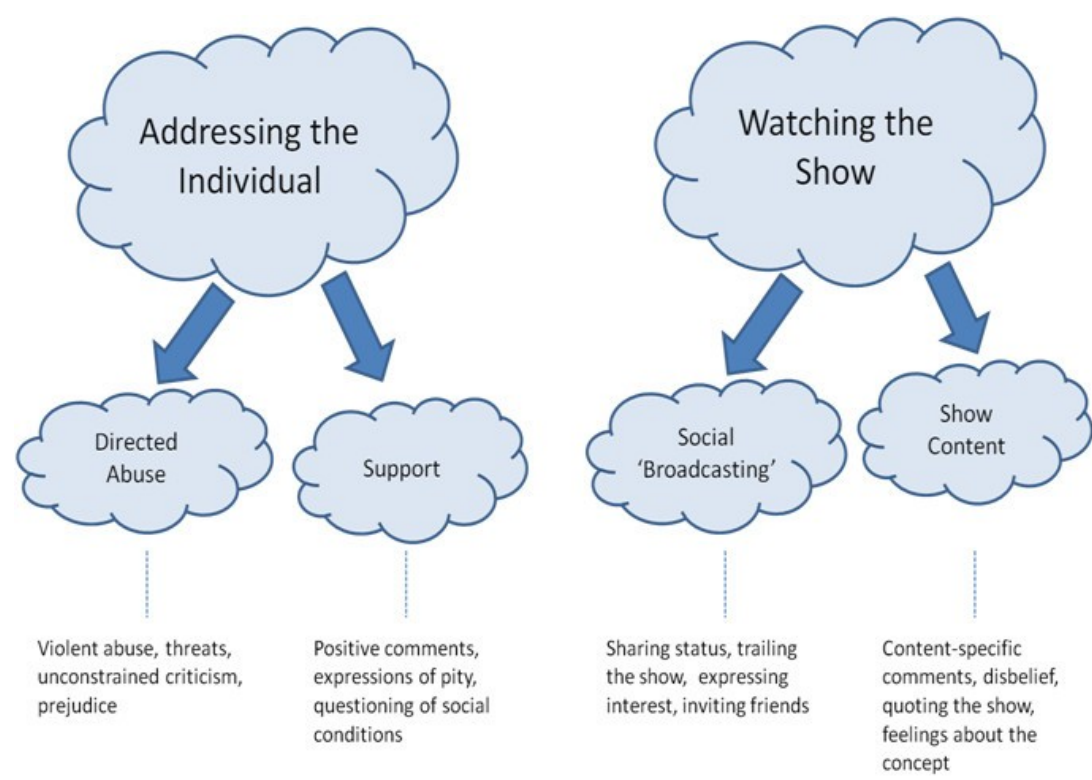

Figure 1: Thematic structure of the tweets tagged with \#ThelmasGypsyGirls

The SentiStrength application [26] was used to perform an assessment of the sentiment polarity of the tweet messages gathered with the \#Thelmas GypsyGirls hashtag and also the corpus of general tweets. The figures in Table 1 represent an average sentiment level for each dimension for each tweet corpus. They suggest that the second-screen posts on Twitter that were tagged with \#ThelmasGypsyGirls and posted during the broadcast of Thelma's Gypsy Girls contained notably more negative language, and therefore more negative sentiment, than a sample of general tweets. Additionally, the retweeted \#ThelmasGypsyGirls posts also reveal an even higher negative and lower positive sentiment than the main \#ThelmasGypsyGirls corpus; this further suggests that second-screen viewers were agreeing with negative messages and were willing to reiterate this sentiment publicly.

SentiStrength estimates the strength of positive and negative sentiment in short texts, and has been shown to have human levels of accuracy for short social web texts [26]. Two sentiment strengths are reported for each message: from -1 to -5 , or from 'not negative' to 'extremely negative', and from 1 to 5 , or from 'not positive' to 'extremely positive'. The ratings for sentiment are derived by comparing the text to a dictionary or positive and negative terms. Refinement of the score is made by detecting mis-spellings and 'booster' or 'negating' terms. For example, 'Love \#thelmasgypsygirls the insight into the Travellers lives is an eye opener!' returns a score of $(3,-1)$, indicating a strong positive sentiment and no negative results, while the message 'These girls are $f^{* * * * * *}$ idiots \#thelmasgypsygirls' (profanity censorship is added here) returns a result of $(1,-5)$ indicating no positive, but extremely negative sentiment.
Each corpus of tweets was analyzed and overall levels of sentiment were determined. Compared to the general tweet messages, the messages containing \#ThelmasGypsyGirls were reported to have higher levels of negative sentiment and lower levels of positive sentiment as shown in Table 1. The negative dimension of the sentiment analysis was significantly higher than that of the general tweets $(t=$ $11.53, \mathrm{p}<0.01)$ indicating that the negativity measured in the \#ThelmasGypsyGirls tweets was higher than that shown in the sample of general tweets. The positive dimension showed no significant difference between the two $(t=-0.42$, $\mathrm{p}>0.1$ ). In addition, the $207 \mathrm{RT}$ messages which were set aside from the main corpus were also assessed for sentiment strength. By assessing the whole of this group and including the duplicates which are formed when a message is retweeted more than once, an indication of the retweeted sentiment was determined (also shown, in bottom row, in Table 1).

The reasons for the predominantly negative tweet messages are, of course, not able to be determined from this analysis alone. By undertaking a thematic analysis of the tweet messages a better understanding of the context and subjectivity of the content can be reached, rather than the context-limited, quantitative approach of the sentiment analysis.

\section{THEMATIC ANALYSIS}

The approach to the thematic analysis [13] used in this study was an inductive, data-driven method as described by Boyatzis [3] and deployed previously for tweet text analysis by other authors e.g. [14]. The analysis was initiated by taking a split of half (group A, $\mathrm{n}=586$ ) of the 1175 \#ThelmasGypsyGirls tweet messages and reading them all individually. Category codes which described the content 
and/or sentiment of the tweet message were recorded by the researcher as the reading took place. The codes were further refined through rereading and a final list of 32 category codes were identified. Following this process, the second half (group $\mathrm{B}, \mathrm{n}=557$ ) of the tweet messages were allocated to three researchers who used the 32 category codes to categorize each of the tweet messages in this sample.

All of the group B tweets were read by the three researchers and a consensus was reached on the assignment of the category codes. By cross-referencing the category codes with the tweet texts, overall themes were identified. From the analysis and consideration of the data, four first order themes were identified which were collected together into two second order themes. These two themes, "Addressing the Individual" (263/557 tweets, $47.2 \%)$ and "Watching the Show" (248/557 tweets, 44.5\%) formed the root of the majority of the tweet texts observed. The remainder of the tweet messages (46/557 tweets, 8.3\%) was ambiguous and was not assigned to these categories. The relationship of these higher order themes with the first order themes is shown in Figure 1 and discussed below.

\section{Addressing the Individual}

This second order theme reveals of much of the intent and directed comments of the second-screen audience. With its decomposition into two opposing first order themes of directed abuse (210/263 tweets, 79.8\%) and support (53/263 tweets, 20.2\%), the content of the tweets reflect people's reactions to the broadcast as it aired. Tweets coded in this theme specifically mentioned the tweeters own reaction to, and feelings about, the subjects of the show, along with wishes, demands for action and threats. Each of the first order themes are discussed below and typical tweet message contents are used for illustration. For the purposes of privacy, no actual names, user names or unique Twitter ID's are included, whilst insulting or offensive words are removed.

\section{Directed Abuse}

This theme was very evident; it was a repetitive theme in the dataset with 210 out of 557 tweets being attributed to this category $(37.7 \%$ of the total). The tweets coded in this theme typically contained abusive language directed at the girls in the show and, in the main, expressed a Twitter user's dislike for the person or their actions, for example "They've all got bad attitudes \#thelmasgypsygirls" or "These Traveller girls are so violent it makes me sick!", "These Gypsy girls are nuttas!," "These girls wanna act tough and gain respect for themselves but they are so immature it's unreal." Many also contained direct threats or wishes of violence: "I want to $f^{* * * * * *}$ slap these stupid $f^{* * * * * *}$ girls," "WOW some of these girls need a slap to knock them into the real world," "Pretty sure a swift tap with a baseball bat would sort them out ... I'm offering...". Some of the messages were graphic in nature and represent a seemingly disproportionate reaction to broadcast content: "I'd kill the girls on \#thelmasgypsygirls," "I just feel like killing ***”. What was evident was the graphic and seemingly un-self-regulated nature of many such messages, as well as how many of them include a "lol" or "haha", indicating laughter "Lol 19 years old n dnt know ur $A B C$,", "LOOOL these girls are getting upset over the word Virgin and they behave like animals". Many of the messages in this theme were, in fact, quite clearly offensive, and inappropriate for publication, even with censorship applied to the offensive terms used. Many of the tweets contained terms which revealed a hatred and dislike of both the girls featured in the broadcast, and the Traveller culture more broadly.

\section{Support}

In contrast to the directed abuse theme, many contributors to the Twitter stream exhibited support and empathy for the show's participants with 53 out of 557 tweets being credited to this group $(9.5 \%$ of the total). This was seen in different ways - whether it was an expression of pity or regret: "Really really can't stand the fact those girls cannot read or write", "Really angers me that those girls are deprived of an education," "Sooo sad that a 17 year old couldn't even recognise all the letters of the alphabet," or whether it was a positive observation: "I would love to be one of \#ThelmasGypsyGirls omg", "Just want to hug lillyanne on \#thelmasgypsygirls!!". The theme suggests that viewers may have been surprised to learn of some of the customs and practices of a different culture, and such messages of support may have been a reaction to that. Indeed, a number of tweets ( 8 of 557) specifically expressed positive sentiments about Traveller culture, "I wanna be a gypsy," "i shud have been a gypsy then again amount of times $i$ move $i$ may aswell be," "I'd love to be a Traveller for a day," "really don't see what everyone has against gypsy's."

\section{Watching the show}

The tweets coded in this theme were about the interaction of the second-screening viewer with the television show itself, and with fellow second screening audience members. This is encapsulated in the two supporting first order themes of social broadcasting (68/248 tweets, $27.4 \%$ ) and show content (180/248 tweets, $72.6 \%)$.

\section{Social Broadcasting}

This theme (12.2\% of the total) became evident through the observation of tweet messages which were intended for other second-screening viewers or users. Those other users in this context could be friends or followers of the originator, or, more generally, anyone who was monitoring the Twitter stream which was 'tuned' to display tweets with the \#ThelmasGypsyGirls hashtag. By updating personal status to friends, or by microblogging a desire to watch the show, the social aspects of using Twitter as a second-screen application were employed. Some typical tweet messages in this theme were: "Giving \#thelmasgypsygirls a go tonight. Looks quite amusing!" and "Time for Thelma and the girls \#ThelmasGypsyGirls". In some cases the show 
was included as a context to other activity which was separate to viewing the show, such as: " $J_{S}$ Had A Nice Shower Now Watching \#ThelmasGypsyGirls :P!'”. It was interesting to note how there was very little negativity expressed in these tweets; many in fact were positive and upbeat in their content, indicating that viewers were pleased to be watching or about to watch the show and were keen to share this on the public timeline.

\section{Show Content}

The Show Content theme $(32.3 \%$ of the total) was comprised of messages which discussed and commented on what was happening in the show. However, unlike tweets coded as "addressing the individual," they do not specifically mention the tweeters own reactions, wishes and threats. "Bridget needs to win this! She's like the only getting involved! It's like being in high school all over again," "its all kicking off on \#thelmasgypsygirls" "The Travellers are fighting cos she deleted her off bbm." In many cases, these messages were questions; viewers questioned the content of the show and wanted clarification or seemed to be initiating conversation relating to some of the issues raised: "Could they not let Shannon work for free or something? \#ThelmasGypsyGirls". The questions frequently expressed disbelief or surprise for many incidents that occurred during the documentary: "How can a wedding dress weigh more than my actual being?! \#ThelmasGypsyGirls" and "They seriously can't even tell the f****** time? \#Thelmas GypsyGirls".

\section{DISCUSSION}

Whilst our thematic analysis did reveal themes that were positive towards the documentary, it also highlighted a strong theme of directed abuse. Moreover, our sentiment analysis indicated that tweets tagged with \#ThelmasGypsyGirls were significantly more negative in their content than a sample of random tweet messages captured at the same time, and furthermore, that retweeted messages exhibited an even higher negative sentiment. It would seem clear that, for this broadcast episode of the documentary at least, Guitton's framing of an emergent 'dark side of social media' [14] holds true. Specifically, the tweets we analysed displayed elements of aggressive behaviour on the part of the tweeters directed toward people they did not know. Further, it appeared that an ad hoc group was formed during the broadcast that attacked a group who appeared different or weaker. The questions for discussion are why might this be happening, and what are the implications?

\section{Why do individuals take part in the abuse?}

Suler [24] suggests that, when interacting in online contexts, people often display less inhibition and apprehension over breaking social norms than they would in other contexts. This leads to people engaging in behaviour that they would otherwise deem unacceptable, a phenomenon that Suler refers to as the "online disinhibition effect." Suler suggests that the lack of overt social cues in this environment, such as direct feedback from either the wider community or the person or group being targeted allow people to feel that they are engaging in a "hit and run" type of interaction, with little consequence for either themselves or the target of their behaviour. The immediacy of Twitter posting, coupled with the fact that it is unmoderated, makes live-tweeting and second-screening susceptible to such a type of disinhibited behaviour. This disinhibition can manifest itself as abusive or offensive tweet messages directed at members of a particular show, the makers of that show, or other viewers. As has been seen in this research and other popular recent second-screen events (such as the Benefits Street TV show in the UK), Twitter users can amplify (or misunderstand) the sentiments of the show and demonstrate disinhibited behaviour through their postings.

\section{Wider concerns: Othering and fear}

Broadcast television produces controversial and challenging content [17] in an effort to 'stimulate' the viewer. In the case of documentaries such as Thelma's Gypsy Girls, the deliberate systematic and repeated highlighting of Travellers' cultural differences [20] seems provide this challenge and stimulation. Of course, promoting large-scale constructive debate around cross-cultural and societal issues can be useful, particularly when the public do not readily engage in such debate. However, broadcast material also has the potential to promote increased stereotyping, or othering, by the television audience of a particular group depicted in that broadcast. Although Said [22] argues that the practice of othering, and the continuous interpretation and reinterpretation of 'difference', is an essential mechanism for the construction of cultural and societal identity, the term is more typically deployed in contexts where the 'other' are negatively perceived, stigmatised, excluded, marginalised and discriminated against. These contexts range from the existentialist philosophy of de Beauvoir's Second Sex, which designated the Other as female, through to the casual everyday othering of any group or community, including ethnic minorities, immigrants, religious groups, asylum seekers, those on benefits, single parents, sex-offenders, political extremists and bankers. Whereas research in media communications has investigated the role of traditional media in influencing attitudes and behaviours, there are unanswered questions around the role of digital services, and social media in particular, in the facilitation and mediation of the othering of groups or communities. For example, the disinhibited nature of social media communication may facilitate the amplification and normalization of cultural stereotyping in wider society, detrimentally impacting upon social cohesion and wellbeing.

boyd [5] has recently drawn attention to the role that digital media and online activities can have in propagating cultures of fear. Whilst she does not explicitly use the term othering, she highlights the fact that "we fear the things - and people -- that we do not understand far more than the things we do, 
even if the latter are much more risky". More importantly, she points to the change, and potential disruption, that social media brings to the propagation of fear and that "hysteria isn't necessarily from on high, but, rather, all around us." In other words, no longer is fear (or hysteria) delivered solely in a top down manner, e.g. from government and the mainstream media, instead it is present in the user-generated social data streams that we absorb from our encounters with the web, and in particular from social platforms such as Twitter, Facebook, and YouTube. A concrete example of this appears to be the very material that is posted during second-screen discussions of Thelma's Gypsy Girls. boyd's statement that 'technology allows us to see people who are different than us, the very people we are likely to fear' [5] goes some way towards explaining why people make abusive and offensive statements while second-screening.

\section{Implications for broadcasters and for Social TV}

TV shows such as Thelma's Gypsy Girls are carefully planned, edited and positioned to highlight the perceived differences between an audience and the Othered group depicted in the programme. Broadcasters create these programmes in the full knowledge that they will create a great deal of social media interest and discussion. Indeed, it is common practice to show Twitter hashtags on-screen during broadcast. However, as demonstrated by the data set analysed here, online discussion can often display antisocial characteristics which are apparently facilitated by the disinhibited nature of social media communication. The abusive messages identified in this paper are an unwanted side effect of a second-screening activity. While the enriching and enhancing qualities of second-screen discussions have been noted [10], the unregulated and unmoderated nature of a Twitter stream permits the posting of material which would be unacceptable and, in many cases, illegal, if spoken in public or printed for publication.

The implications for television broadcasters and social TV application developers seem clear: the way that people engage in second-screen activities around programming must be carefully designed and promoted in order to discourage abusive, anti-social and illegal behaviour. This task becomes easier the more tightly coupled the social TV application and the broadcast become. Dedicated secondscreening applications or social TV screen overlay systems, such as the $4 N o w^{5}$ app, have the ability to monitor and filter the message streams that they display, while more loosely coupled systems, such as Twitter, afford less opportunity to do so. In addition, perhaps new applications can be developed with the intention of undermining the online

\footnotetext{
5 See "Channel 4 second-screen app 4 Now to allow real-time viewer interaction" Article in The Guardian (5 June 2013)

"http://www.theguardian.com/media/2013/jun/05/channel-4-secondscreen-app-4now"
}

disinhibition effect, such as removing the likelihood of anonymous messages.

Broadcasters should also consider how they publicise a Twitter hashtag as the 'preferred' tag for the second-screen discussion. The publication of a hashtag on-screen encourages discussion of that programme on Twitter. However, the conversation is not then controlled or moderated by the broadcaster. Broadcasters must consider whether they are, in any way, responsible for reactions to their programming on social media sites. If broadcasters were seen as encouraging the abuseive posts identified here, they may be open to future criticism by the public, advertisers and regulatory bodies.

\section{CONCLUSION}

This paper contributes to the understanding of how users engage in second-screen discussion whilst viewing broadcast television. We present an investigation into the nature of Twitter-facilitated second-screening posts relating to Thelma's Gypsy Girls, one of a series of controversial documentary programmes portraying the Irish Traveller community that have recently been aired by the UK publicservice television broadcaster Channel 4. Sentiment analysis highlighted the general negativity of these posts whilst a detailed thematic inquiry revealed the often abusive and aggressive messages aimed directly at the community and individuals portrayed in the broadcast material. We suggest that second-screening and live-tweeting alongside broadcast television generates new concerns about online abuse. Specifically, the nature of social media interactions, being asynchronous, anonymous, and lacking in direct feedback, lowers people's inhibitions about engaging in abusive and anti-social behaviour. We argue that this disinhibited behaviour, when directed towards characters on a TV programme, particularly where those characters are portrayed as different to the social norms of the audience, can facilitate stereotyping, othering and prejudice in wider society. Television broadcasters and social TV application developers have a responsibility to be aware of these dangers and to act so as to minimize the impact upon social cohesion and wellbeing.

\section{REFERENCES}

1. Atkinson, C. The Backchannel: How Audiences Are Using Twitter and Social Media and Changing Presentations Forever, New Riders, 2010.

2. Broadcasters' Audience Research Board, (2012) http://www.barb.co.uk/

3. Boyatzis, R., Transforming Qualitative Information: Thematic Analysis and Code Development, Sage Publications, 1998.

4. boyd, d., Lotan, G., Tweet, Tweet, Retweet: Conversational Aspects of Retweeting on Twitter, 43rd Hawaii International Conference on System Sciences (HICSS), 5-8 January, 2010. 
5. boyd, d., The Power of Fear in Networked Publics. SXSW. Austin, Texas, March 10, 2012.

6. Bruns, A., Burgess, J., (2011) The use of Twitter hashtags in the formation of ad hoc publics. In 6th European Consortium for Political Research General Conference, 25 - 27 August 2011, University of Iceland, Reykjavik

7. Caspi, A., Gorsky, P., Online Deception: prevalence, motivation and emotion, CyberPsychology \& Behavior. February 2006, 9(1): 54-59.

8. Cesar, P., Bulterman, D., Jansen, A., Usages of the Secondary Screen in an interactive television environment: Control, enrich, share and transfer television content, Changing Television Environments, Lecture Notes in Computer Science, 2008, Volume 5066/2008, 168-177.

9. Chen, L., \& Chen, T. L. (2012). Use of Twitter for formative evaluation: Reflections on trainer and trainees' experiences. British Journal of Educational Technology, 43(2), E49-E52.

10.Doughty, M., Lawson, S., Rowland, D., Co-viewing live TV with digital backchannel streams, EuroITV'11 Proc. 9 th international interactive conf. on Interactive Television Pages 141-144 ACM New York, NY, USA.

11.Doughty, M., Rowland, D., Lawson, S. (2012). Who is on your sofa?: TV audience communities and second screening social networks. In Proceedings of the 10th European conference on Interactive tv and video (EuroiTV '12). (pp. 79-86) ACM, New York, NY, USA.

12. Evangelia Mantzari, George Lekakos, and Adam Vrechopoulos. 2008. Social TV: introducing virtual socialization in the TV experience. In Proc. of the 1st International Conference on Designing Interactive User Experiences for TV and Video, 2008.

13.Fereday, J., Muir-Cochrane, E., Demonstrating Rigor Using Thematic Analysis: A Hybrid Approach of Inductive and Deductive Coding and Theme Development, Int. J. of Qualitative Methods, 5(1), 2006.

14. Guitton M.J. (2014) The importance of studying the dark side of social networks. Computers in Human Behavior 31 (2014) 355.

15. Jamison-Powell, S., Linehan, C., Daley, L., Garbett, A., Lawson, S. 2012. "I can't get no sleep": discussing \#insomnia on twitter. In Proc. 2012 ACM annual conference on Human Factors in Computing Systems (CHI 2012). ACM, New York, NY, USA, 1501-1510.
16. Jay, T., \& Janschewitz, K. (2008). The pragmatics of swearing. $J$. of Politeness Research. Language, Behaviour, Culture, 4(2), 267-288.

17. Jensen, T, Ringrose, J., Feminist Media Studies (2013): Sluts that Choose Vs Doormat Gypsies: Exploring the Affect in the Postfeminist, Visual Moral Economy of My Big Fat Gypsy Wedding, Feminist Media Studies

18. Joinson, A., Disinhibition and the Internet, in Psychology and the Internet: Intrapersonal, Interpersonal, and Transpersonal Implications, ed. Gackenbach, J., 2007, Elsevier Press, Burlington, MA.

19. Kowalski, R., Limber, S., Agatston, P., Cyberbullying: Bullying in the Digital Age, $2^{\text {nd }}$ ed. John Wiley \& Sons, 2012.

20. Richardson, J. \& O'Neill, R., 'Stamp on the Camps': the social construction of Gypsies and Travellers in media and political debate, in Gypsies and Travellers: Empowerment and Inclusion in British Society, eds. Richardson, J. \& Ryder, A., The Policy Press, 2012.

21. Riggins, S. H. (1997). The rhetoric of othering. The language and politics of exclusion: Others in discourse, 1-30.

22. Said, E. (1978). Orientalism (New York. Pantheon, 6, 14-27.

23. Schirra, S, Sun, H., \& Bentley, F. (2014) Together Alone: Motivations for Live-Tweeting a Television Series, to appear in Proc CHI 2014. ACM Press.

24. Suler., J., The Online Disinhibition Effect, CyberPsychology \& Behavior. June 2004, 7(3): 321326.

25. O'Sullivan, P., Flanagin, A., Reconceptualizing 'flaming' and other problematic messages, New Media \& Society March 2003 vol. 5 no. $169-94$

26. Thelwall, M., Buckley, K., Paltoglou, G., Cai, D., Kappas., A., Sentiment strength detection in short informal text, J. American Society for Information Science and Technology 61(12) 2010, 2544-2558

27.Thelwall, M., Buckley, K., Paltoglou, G., Sentiment in Twitter events, J. American Society for Information Science and Technology, 62(2), 2011, 406-418.

28. Tremlett, A., (2013) Demotic or demonic? Race, class and gender in 'Gypsy' reality TV. to appear in The Sociological Review.

29. Vandebosch, H., \& Van Cleemput, K. (2009). Cyberbullying among youngsters: Profiles of bullies and victims. New Media \& Society, 11(8), 1349-137 